Article

\title{
Bulk Surfaces Coated with Triangular Silver Nanoplates: Antibacterial Action Based on Silver Release and Photo-Thermal Effect
}

\author{
Agnese D'Agostino ${ }^{1}$, Angelo Taglietti ${ }^{1, *}$, Roberto Desando ${ }^{1}$, Marcella Bini ${ }^{1}$, \\ Maddalena Patrini ${ }^{2}$, Giacomo Dacarro ${ }^{1,2}$, Lucia Cucca ${ }^{1}$, Piersandro Pallavicini ${ }^{1}$ \\ and Pietro Grisoli ${ }^{3}$ \\ 1 Department of Chemistry, University of Pavia, Viale Taramelli 12, 27100 Pavia, Italy; \\ agnese.dagostino01@universitadipavia.it (A.D.); desandoroberto.91@hotmail.it (R.D.); \\ marcella.bini@unipv.it (M.B.); giacomo.dacarro@unipv.it (G.D.); lucia.cucca@unipv.it (L.C.); \\ psp@unipv.it (P.P.) \\ 2 Department of Physics, University of Pavia, Via Bassi 6, 27100 Pavia, Italy; maddalena.patrini@unipv.it \\ 3 Department of Drug Sciences, University of Pavia, Viale Taramelli 14, 27100 Pavia, Italy; \\ pietro.grisoli@unipv.it \\ * Correspondence: angelo.taglietti@unipv.it; Tel.: +39-342-987-342
}

Academic Editor: Guogang Ren

Received: 2 November 2016; Accepted: 30 December 2016; Published: 6 January 2017

\begin{abstract}
A layer of silver nanoplates, specifically synthesized with the desired localized surface plasmon resonance (LSPR) features, was grafted on amino-functionalized bulk glass surfaces to impart a double antibacterial action: (i) the well-known, long-term antibacterial effect based on the release of $\mathrm{Ag}^{+}$; (ii) an "on demand" action which can be switched on by the use of photo-thermal properties of silver nano-objects. Irradiation of these samples with a laser having a wavelength falling into the so called "therapeutic window" of the near infrared region allows the reinforcement, in the timescale of minutes, of the classical antibacterial effect of silver nanoparticles. We demonstrate how using the two actions allows for almost complete elimination of the population of two bacterial strains of representative Gram-positive and Gram-negative bacteria.
\end{abstract}

Keywords: antibacterial surfaces; silver nanoplates; seed-growth synthesis; photothermal effect; hyperthermia

\section{Introduction}

Functional nanomaterials are increasingly finding wide success in several research fields devoted to human well-being. Just to name a few recent examples, functional nanomaterials can be used for nanodelivery of anticancer drugs [1] and, more generally, of pharmaceuticals molecules [2], for theranostic purposes in the presence of peculiar plasmonic effects given by properly functionalized noble metal nano-objects [3]. Functional nanomaterials can even to provide new solutions to energy production-related problems, as in the case of catalytic nanoarchitectonics [4].

Recent news [5] about the existence of an antibiotic-resistant Escherichia coli superbug spread concern throughout the whole biomedical scientific community, but also in media and the general population. Pan-drug-resistant bacteria can be particularly dangerous when infecting materials used in the nosocomial environment: bacterial and biofilm-associated infections involving medical, surgical, and prosthetic surfaces are probably the leading cause of severe nosocomial problems [6,7]. In situations where conventional antibiotics are not able to eliminate bacteria from surfaces, a possible tool is the coating of surfaces with silver nanoparticles (AgNPs), acting as a very efficient antimicrobial agent and representing an alternative to antibiotics [8]. The mechanism of this antibacterial action is 
still under debate, but it is now accepted that it involves the release of $\mathrm{Ag}^{+}$ions and their subsequent interaction with bacteria, even if one cannot exclude the presence of a short-distance nanomechanical action caused by the direct interaction of AgNPs with the bacterial cell membrane [9]. Usually, AgNPs are much more effective towards Gram-negative (Gram-) bacterial strains than towards Gram-positive $(\mathrm{Gram}+)$, due to the fact that Gram+ bacteria present a relatively thick $(20-80 \mathrm{~nm})$ and continuous cell wall almost entirely made of peptidoglycan, while Gram - bacteria feature a less thick, less solid, and less resistant peptidoglycan layer $(5-10 \mathrm{~nm})$ surrounded by a phospholipidic membrane [10].

While a huge number of papers report the antibacterial effects of spherical AgNPs, until a few years ago, a limited number of studies investigating the antibacterial activity of anisotropic silver nano-objects had appeared. A first fundamental report [11] showed that truncated triangular nanoplates were more efficient than spherical AgNPs in killing E. coli, suggesting the existence of a shape-dependent interaction between AgNPs and Gram- bacteria. Recently, the use of anisotropic silver nanoparticles has started to raise a greater interest, driven also by the fact that localized surface plasmon resonance (LSPR) properties of noble metal nano-objects are strongly dependent on size and shape, and that this feature can be exploited to obtain nano-objects having a wide range of colors, a feature which is exploitable in the coating of textile fibers to obtain colorful antibacterial fabrics $[12,13]$. Recent studies have discussed the antibacterial effects of triangular, truncated triangular and hexagonal plates, and some comparison with spherical AgNPs was attempted [14,15]. In a very recent example, the strong antibacterial activity of anisotropic nanoparticles toward Pseudomonas aeruginosa cells was demonstrated when applied to corneal replacements [16].

Interestingly, noble metal nanoparticles can be used for antibacterial purposes by also exploiting a different feature: the hyperthermia produced by their photo-thermal effect [17]. Gold nanorods (GNRs) [18] and gold nanostars (GNSs) [19] have been used to this purpose. Even if gold is not an "antibacterial element", gold nano-objects of anisotropic shape-featuring two (or more) intense LSPR bands-can achieve thermal relaxation after proper irradiation. This produces hyperthermia in their surroundings, which can be used to kill bacteria. When the LSPR band falls in the near-IR range "therapeutic window" (NIR, 750-950 $\mathrm{nm}$ ) where tissues and blood are semi-transparent, laser irradiation can be used. One can thus imagine applying this strategy to the treatment of possible infections on subcutaneous devices, if they are coated with these kind of nanomaterials. In a recent example, we used a monolayer of GNSs grafted on bulk glass samples [20] to have a model of an active surface. Laser irradiation of this monolayer, exploiting the NIR LSPR absorption of GNSs, yielded an efficient photo-thermal conversion causing hyperthermia, which efficiently kills bacteria cells in Staphylococcus aureus biofilms.

The ability to obtain an efficient photo-to-heat conversion is not only limited to gold nano-objects. In two recent and pioneering examples [21,22], the photo-thermal effect given by silver nanoplates was smartly investigated for photo-thermal treatments of cancer cells when irradiated at $800 \mathrm{~nm}$, a wavelength value where a plasmonic band can be easily placed by tuning morphology and dimensions of the objects themselves [23]. Flat silver nano-objects typically show an in-plane dipole plasmon resonance band, whose position can be placed in the NIR region and tuned at a desired value, just by regulating the dimensions and the aspect ratio of the objects [24].

Few recent papers have been dedicated to bimetallic nanostructures in which the photo-thermal effects have been studied for augmenting the release of silver, followed by evaluation of this increased release for microbicidal tasks [25-27]. Thus, it seems quite odd that no examples are reported in literature on the use of the photo-thermal effects of pure anisotropic silver nanoplates for antimicrobial applications. Only very recently, we have proposed a unique approach, which consisted of growing almost flat silver nanostructures directly on a layer of small AgNP seeds, already grafted on glass. The approach provides for a certain degree of control of the dimensions of the objects obtained, resulting in a quite high surface density of silver (about $6.2 \mu \mathrm{g} / \mathrm{cm}^{2}$ ) and exploitation of the antibacterial activity achieved by silver release and photo-thermal features [28]. 
Here, we were interested in a different approach, in order to obtain a more general preparation route. The simple idea is to synthesize anisotropic silver nano-objects with the desired features (shape, dimension, LSPR features, type of coating), and then graft them on a bulk surface. This last step can be easily performed by dip-coating glass samples (properly silanized, introducing a grafting function) in the silver nanoplates colloidal solutions. Once again, we chose glass as an economic material with wide applicability.

Triangular silver nanoplates can be synthesized in a plethora of ways [23]. In general, anisotropic noble metal nano-objects are prepared by seed-growth methods, and these preparative strategies usually exploit surfactant molecules to produce and direct the anisotropic growth $[29,30]$. Unfortunately, it can be hard to remove these capping molecules from the nanoparticles surfaces, and this often complicates the chemistry that is needed for further coatings [31]. Also, molecules like surfactants may have a toxic effect on human cells, and this of course is a main issue when nano-objects, in colloidal suspension or grafted on a solid surface, are intended to be used in applications involving human health [32]. Even the presence of polymeric stabilizers, which can improve the growth as well the stability of anisotropic particles [24,33], can represent a problem. Polymeric stabilizers could affect the antibacterial activity [9]—interfering with silver nanoparticle surface oxidation, a step which is needed for subsequent silver ion release [34] — as well their grafting onto surfaces, which can be strongly dependent on the nano-object's surface charge. On the other side, the citrate anion is not harmful and, when used in seed growth synthesis [23,24,28,29], preferentially binds to (111) facets of growing particles, slowing down the vertical expansion and thus allowing anisotropic growth, producing nanoplates. Moreover, it ensures a negative surface charge, which is crucial for grafting to bulk surfaces functionalized with amines, which are protonated when used in water at neutral or slightly acidic conditions.

With these considerations in mind, we prepared bulk glass surfaces coated with trimethoxysilylpropyl (polyethylenimine) (PEI-silane, see Scheme 1) — a polymer containing primary, secondary, and tertiary amines-and to these surfaces we grafted opportunely synthesized citrate-coated silver nanoplates to obtain a prototype of a medical device surface [34] carrying a layer of silver nanoplates with the ability to strongly absorb laser radiation in the NIR range. We demonstrate that this coating exerts an antibacterial activity relying on: (i) the well-known microbicidal activity typically obtained with release of silver ions from nano-objects; (ii) the antibacterial action caused by the hyperthermia produced by the photo-thermal effect.

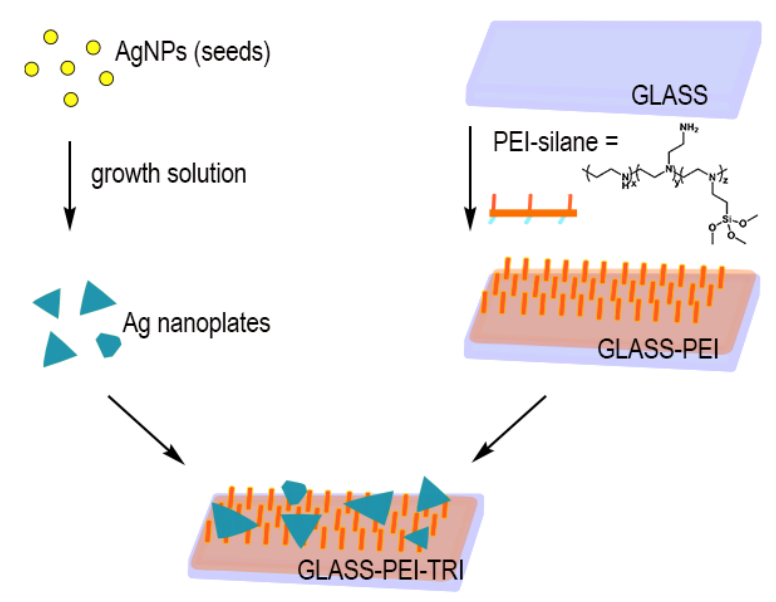

Scheme 1. A schematic representation of the synthetic route to polyethylenimine (PEI)-functionalized glass with citrate-capped Ag nanoplates (GLASS-PEI-TRI) samples. 


\section{Results and Discussion}

\subsection{Nanoplates Synthesis}

As already stated, we opted for a synthetic route based on a seed-growth method, but avoided any surfactant or polymer in an attempt to produce the nanoplates in absence of any potentially harmful reactant, as well to pursue an economic and "green" preparation and to obtain "safe" silver nano-objects with potential antibacterial action.

Seeds were prepared by modifying a reported method [35], which yielded small spherical nano-objects of about $5 \mathrm{~nm}$ diameter. Spectrum of the colloid shows the expected narrow LSPR band centered at about $396 \mathrm{~nm}$. In order to standardize the preparation, seeds were diluted such that stock seed solutions with the LSPR band had an absorbance of 0.5 units.

The plates were prepared simply by adding a precise volume of seeds at a proper volume of growth solution, which was composed only by the properly optimized amounts of ascorbic acid, sodium citrate, and silver nitrate. A slow change in suspension color was noted, corresponding to growth of an intense LSPR band, which shifted towards NIR over time while increasing its intensity. The phenomenon was completed in less than $12 \mathrm{~h}$, and no further sensible changes of LSPR features were observed at the time scale of one week, indicating the stability of the obtained colloidal suspension. Completion of nanoplates' growth is also indicated by the complete disappearance of the UV absorption band of ascorbic acid, centered at $266 \mathrm{~nm}$. The synthesis is quite reproducible when the quantity of seed is strictly controlled, yielding colloidal suspensions where three bands are clearly recognized [33]: as shown in Figure 1a, there is one band at $330 \mathrm{~nm}$ (which can be ascribed to the out-of-plane quadrupole resonance for triangular nanoplates), one at about 420-440 $\mathrm{nm}$ (the zone where the out-of-plane dipole resonance is usually found), and one that is more intense-the in-plane dipole resonance-at about $845 \pm 30 \mathrm{~nm}$ (average value based on eight preparations). A spectrum obtained for a representative standard preparation is reported in Figure 1a, while four spectra coming from four repetitions of the identical standard preparation are reported in the Supplementary Material (Figure S4), accounting for an acceptable degree of reproducibility.
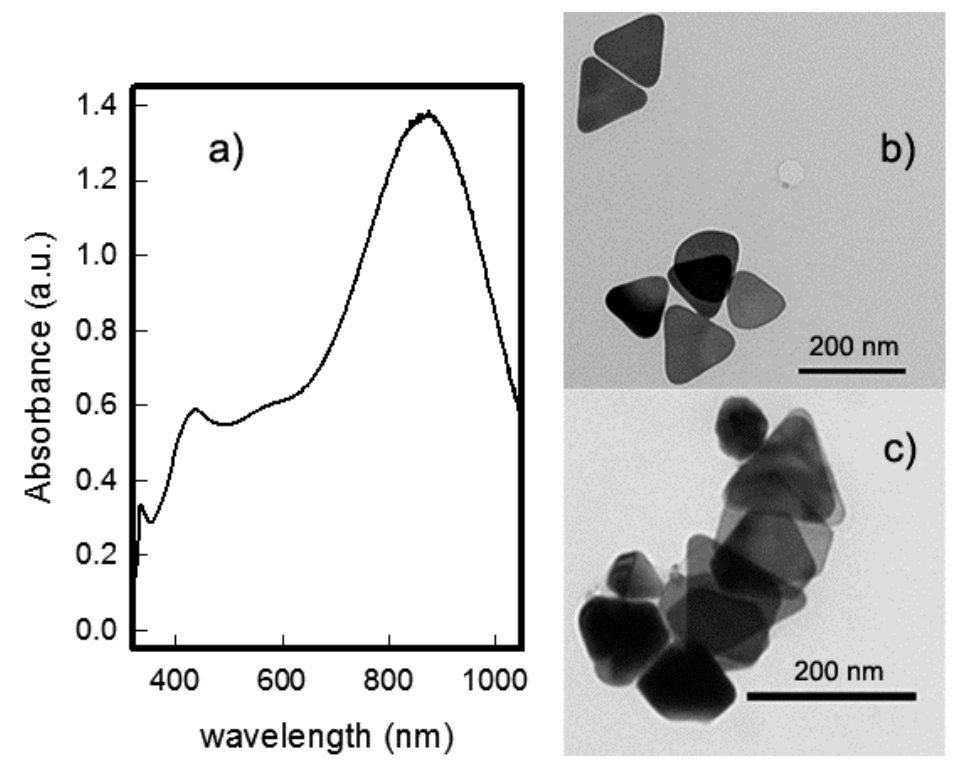

Figure 1. (a) UV-vis spectra of colloidal suspension of silver nanoplates obtained with the standard preparation; (b,c) Transmission electron microscope (TEM) images of silver nanoplates obtained with the standard preparation. 
It is interesting to note that this last LSPR band can be roughly adjusted by changing the quantity of seed solution added: reducing the seed quantity by $33 \%$ results in an LSPR band shifting toward $900 \mathrm{~nm}$, while increasing the added seed quantity to double that of the standard value shifts the final LSPR band close to $800 \mathrm{~nm}$ or less. As is obvious, the presence of smaller quantities of seeds produces larger objects with the in-plane dipole resonance moved to higher wavelengths, while the addition of a higher quantity of seeds, when the amount of silver ion precursor and other reactants are kept constant, produces smaller objects with the in-plane dipole band shifted to lower wavelengths.

The complexity of the spectra and the broad absorption band (in the range of 400-650 nm), indicates the presence of several type of different objects.

As shown in Figure 1b,c, transmission electron microscope (TEM) analysis of the suspensions obtained with the standard preparations yielding an in-plane dipolar LSPR placed at $845 \pm 30 \mathrm{~nm}$ confirms the presence of a majority of triangular plates, with various degrees of snipping of the corners: objects range from almost regular triangles with slightly rounded corners, to a small number of almost hexagonal plates, as can be seen in Figure 1b,c. A certain number of smaller, more rounded objects, as well of objects of other shapes, can be noticed (TEM images in Supplementary Material, Figures S6 and S7). From a few of the objects which were placed vertically in agglomerates observed in TEM images (see Supplementary Material, Figure S6) we estimated an average thickness of about $20 \mathrm{~nm}$. The dimensions of regular triangles obtained with the standard preparation were measured, calculating (as a qualitative parameter for each triangular plate) the three altitude values (defined as the distance between a vertex of a triangle and the opposite side at right angles) and obtaining an average value (coming from four preparations) of $120 \pm 25 \mathrm{~nm}$. A histogram representing the distribution of the average altitude value for 43 regular triangles obtained from TEM images coming from the standard preparation reported in Figure 1a is shown as an example in Figure S8 in the Supplementary Material.

As already deduced from LSPR spectra, changing the quantity of seed solution added produces a variation in the dimensions of nanoplates obtained, without altering the mean morphology of the objects. Reducing the seed quantity ( $33 \%$ compared to the quantity used in the standard preparation) results in an enlargement of objects (see TEM images reported in Supplementary Material, Figure S9), with regular triangles' average altitudes reaching up to $200 \mathrm{~nm}$. Increase of the seed quantity (to double that of the quantity used in the standard preparation) produces smaller objects (with average altitudes of regular triangles reduced to about $80-100 \mathrm{~nm}$ ), as can be seen in the TEM images reported in Figure S10 in Supplementary Material. In both cases, changes in the morphologies of the obtained objects in the colloids were not observed.

We must stress that it was not the aim of this work to study the effects of the fine variations of synthetic parameters (such as the quantity of seeds added) on nanoplates' size and morphology and on the distribution of object shapes. We simply performed a rapid screening, and we were satisfied to find the synthetic conditions needed to produce colloidal suspensions with an intense in-plane dipolar LSPR at the desired wavelength value. The preliminary synthetic investigation shown here should be expanded and described more accurately, and this topic is actually the subject of a more detailed investigation in our laboratory.

Measurements of the $\zeta$-potential of the colloid gave a value of $-40.6 \pm 4.5 \mathrm{mV}$, suggesting quantitative coating of the objects with citrate and explaining the high stability of the suspensions, as citrate provides enough surface charge to stabilize particles against aggregation. Stability was also demonstrated by the fact that the colloidal suspension of nanoplates can be centrifuged at $5000 \mathrm{rpm}$ for $15 \mathrm{~min}$ and then resuspended in water without any visible change in LSPR spectra. The role of citrate as a stabilizing agent with a shape-directing action is well known: citrate anions preferentially bind to (111) facets of the crystals, which grow after the seed addition to the growth solution, slowing down the vertical expansion and thus obtaining anisotropic growth of the nanoplates. This was also confirmed by X-ray diffraction (XRD) patterns (see Supplementary Material, Figure S15) obtained from a sample of the colloidal suspension. The suspension was centrifuged, concentrated, deposited on blank glass, and dried, obtaining a spot of solid powder of nanoplates. Only the (111) reflection of a 
face-centered cubic (FCC) structure is observed, indicating that the basal lattice plane of nano-objects is (111), as expected from the growth-directing action of citrate [23].

It is important to stress the fact that a rough but (for our purposes) useful control on the plates' morphology and dimensions was obtained using only ascorbate and citrate, and by controlling the quantity of seeds. In absence of surfactant or polymeric stabilizers, we obtained a colloid with an LSPR spectrum that fits our need for intense NIR absorption in the so-called "therapeutic window". In addition, citrate ensures a high negative charge on nanoplates, which is crucial for colloidal stability and for interaction with protonated amines (which will be present on bulk glass surfaces as a grafting layer), while avoiding the use of harmful coating agents. Moreover, AgNPs prepared in presence of citrate usually show very efficient antibacterial action [9,10,34-36], a fact which is not ensured in presence of coating agents that could interfere with the release of silver ions.

\subsection{Grafting of Nanoplates: Preparation and Characterization of Polyethylenimine (PEI)-Functionalized Glass with Citrate-Capped Ag Nanoplates (GLASS-PEI-TRI) Samples}

The well-known antibacterial action of AgNPs can be easily brought to macroscopic scale. Recently, we performed this task in several ways; for example, by creating a firmly grafted AgNPs monolayer on glass (used as a model surface), exploiting the Ag-S bond after obtaining a -SH layer on bulk glass by means of the formation of a self-assembled monolayer (SAM) of mercaptopropyltriethoxy silane (MPTS) [34]. We obtained similar results using electrostatic interactions between ammonium groups and negative charges present on AgNPs surfaces: a monolayer of citrate-capped AgNPs can be obtained through preliminary silanization of glass surfaces with 3-aminopropyltriethoxysilane (APTES), [36]. The same can be done preparing a layer of PEI-silane on glass as an adhesive layer [37]. In all these cases, we were able to obtain excellent stability of the nano-objects' layer in aqueous media, with prolonged release of silver ions, controlled local concentration of $\mathrm{Ag}^{+}$without any detachment of AgNPs, and a strong antibacterial action with limited risks towards human health. Here, we followed the same approach, and prepared a PEI-terminated surface ready to graft silver nanoplates, which are negatively charged ( $\mathrm{pH}$ of colloidal suspension is around 6 in these conditions, with a distinctly negative $\zeta$-potential as reported in the previous paragraph), because of citrate coating. PEI-silane (see Scheme 1) is a polymer composed of an average of 3.6 propyltrimethoxysilane groups and 56 ethylenimine monomers (i.e., 56 amine moieties (including primary, secondary and tertiary amines). Each polymer molecule has multiple alkoxysilane attaching points. In the standard procedure, $21 \times 26 \mathrm{~mm}$ glass slides were cleaned and activated with piranha solution, producing an $-\mathrm{OH}$ rich, highly hydrophilic surface, having a contact angle $<10^{\circ}$ and ready to react quickly with PEI-silane in ethanolic solution, yielding PEI-functionalized surfaces (GLASS-PEI). As a characterization step, static contact angle $\left(49^{\circ} \pm 4^{\circ}\right)$ was measured after functionalization, in good agreement with our previous results [28,37].

Grafting of nanoplates was obtained by dipping the GLASS-PEI slides for $14 \mathrm{~h}$ in aqueous colloidal suspensions of citrate-capped Ag nanoplates. The typical UV-vis-NIR spectra of glass samples obtained after this treatment (GLASS-PEI-TRI), reported in Figure 2a, clearly shows the grafting of the nanoplates. The out-of-plane dipole band in the zone around $400-450 \mathrm{~nm}$ can be recognized. The in-plane dipolar band is centered in the $820 \pm 30 \mathrm{~nm}$ range, with a $20-30 \mathrm{~nm}$ blue-shift with respect to the starting colloidal suspension. This shift is essentially due to the decrease of the local refractive index $(n)$ on passing from water $\left(n_{\text {water }}=1.3339\right)$ to glass and air at the interface $\left(n_{\text {air }}=1.0003\right)$ [34]. The out-of-plane quadrupole resonance band observed at $330 \mathrm{~nm}$ cannot be found, probably because it is too weak and covered by the absorbance of glass itself in the UV region. Once again, broadening of the LSPR bands can be explained by the presence of objects with different morphologies and dimensions. Visually, the samples show a very light grey-blue color. 

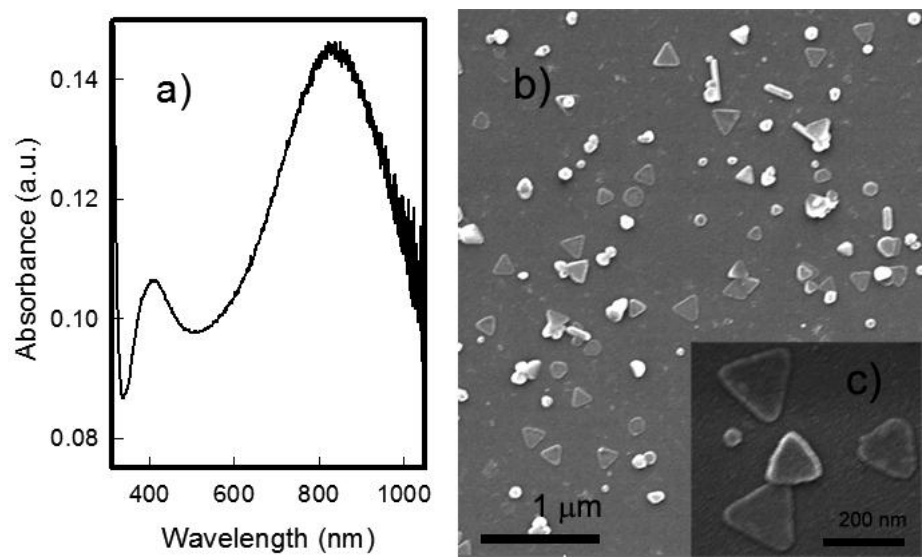

Figure 2. (a) UV-vis spectra of a GLASS-PEI-TRI sample; (b) Scanning electron microscope (SEM) image of a GLASS-PEI-TRI sample; (c) detail of a high magnification SEM image showing the presence of triangular nanoplates on the GLASS-PEI-TRI samples.

Figure 2 also shows a couple of scanning electron microscope (SEM) images for GLASS-PEI-TRI samples at different magnifications. As can be seen in the image reported in Figure $2 b$, a homogeneous distribution of all the objects already observed in the TEM images was obtained, with the presence of some agglomerations of objects that still retain dimension and morphology. This can be more precisely observed in Figure 2c, where some regular triangles are shown. The homogeneous and well-spaced distribution on the surface allowed us to calculate the composite percent of different objects obtained in the seed-growth synthesis (already noticed in TEM images) and grafted on glass with this last step. Counting the objects found in large area SEM images gave the following constant composition: $49 \%$ of regular triangles, $19 \%$ of triangles with various degrees of snipping (up to hexagonal shape), $12 \%$ of rod-shaped objects, and $20 \%$ of more rounded, discoidal objects. Size measurement of several regular triangles gave a value of $125 \pm 25 \mathrm{~nm}$ for their altitude, in good agreement with the values derived from TEM.

The preparation of bulk glass samples coated with silver nanoplates is quite reproducible: in Figure S11, spectra of four samples coming from four identical (but obtained in four different reaction vessels) preparations obtained from the same colloidal suspension are reported, while Figure S12 reports the spectra of seven samples obtained in a single preparation (i.e., in the same reaction vessel). GLASS-PEI-TRI slides are very stable in air (i.e., their spectra did not change significantly for at least a 2-month period).

XRD patterns of GLASS-PEI-TRI samples showed once again only the presence of a (111) peak. In this case, a certain broadening is observed when the peak is compared to the one found in the case of the nanoplates powder (see Figure S15 in Supplementary Material). This broadening can be explained on the basis of the relatively low surface concentration of plates in the GLASS-PEI-TRI samples.

We also carried out the characterization of the GLASS-PEI-TRI with quantitative oxidation (using controlled amounts of strong acid) of the silver nanoparticles that had been grafted on the samples, and analyzed the obtained solution by means of inductively coupled plasma (ICP). Data from eight different samples, coming from eight different preparations, gave an average of $1.87(0.30) \times 10^{-6} \mathrm{~g} / \mathrm{cm}^{2}$ of silver. This quantity is higher than those found for small spherical AgNPs monolayer samples $[34,36,37]$, but sensibly lower than the described case in which a seed-growth synthesis of flat silver objects is performed directly on glass [28].

A feature of primary interest for the applications we have in mind is the ability to release a controlled quantity of silver without detachment of nano-objects in the environment, when the sample is immersed in an aqueous environment. We investigated this aspect by placing freshly prepared GLASS-PEI-TRI samples in ultrapure water for $48 \mathrm{~h}$, and then measuring their UV-vis-NIR spectra and examining their SEM images (see Figures S13 and S14 in Supplementary Material). We also 
measured the UV-vis-NIR spectra of obtained solutions, which were subsequently analyzed with ICP. Data from the solutions obtained after $48 \mathrm{~h}$ immersion in ultrapure water of GLASS-PEI-TRI samples showed the presence of $0.075(0.030) \times 10^{-6} \mathrm{~g} / \mathrm{cm}^{2}$ of silver. UV-vis-NIR spectra on the same solutions did not show any LSPR absorbance, thus ruling out the presence of plasmonic nano-objects and detachment of objects from the surfaces. Consequently, all the silver found in solution is ionic, and not belonging to detached metallic nano-objects. This was confirmed from SEM images on the samples extracted from the solutions after the immersion, which showed the same homogeneous distribution of the objects that was observed in the freshly prepared samples, with objects conserving their average number, morphology, and size; this can be observed by comparing Figure 2 and Figure S14. Moreover, the UV-vis-NIR spectra of the same GLASS-PEI-TRI samples are almost identical to the spectra registered before the immersion. Even if the situation in more complex media is sensibly different, these experiments indicate that: (i) the objects are stably grafted on the surfaces; (ii) there is a limited release of silver ions from these objects, which (compared to the total quantity of silver found on the samples) means that only about $4 \%$ of total grafted silver is released after $48 \mathrm{~h}$ of immersion in water; and (iii) this release neither significantly changes the shape and dimensions of the objects, nor their consequent LSPR features.

At this stage, we wanted to check the photo-thermal features of the grafted nano-objects (i.e., their ability to produce localized hyperthermia when properly irradiated). To do this, GLASS-PEI-TRI samples showing an LSPR maximum around $830 \mathrm{~nm}$ were irradiated with a laser source at an $808 \mathrm{~nm}$ with a power of $200 \mathrm{~mW}$, using a spot of $1.0 \mathrm{~cm}$ in diameter, corresponding to an irradiance of $0.26 \mathrm{~W} / \mathrm{cm}^{2}$. Using a thermocamera, the temperature in correspondence to the irradiated spot was measured and plotted against time of irradiation. As can be clearly seen in Figure 3, irradiation causes a temperature increase of about $11-12{ }^{\circ} \mathrm{C}$ in a few seconds. The same behavior is observed when the measure is repeated on a sample after $48 \mathrm{~h}$ of immersion in water, demonstrating once again that a prolonged immersion in water does not produce any change in plasmonic features of the nanoplates layer. As is predictable, irradiation of uncoated glass samples in the same conditions does not produce any measurable hyperthermia.

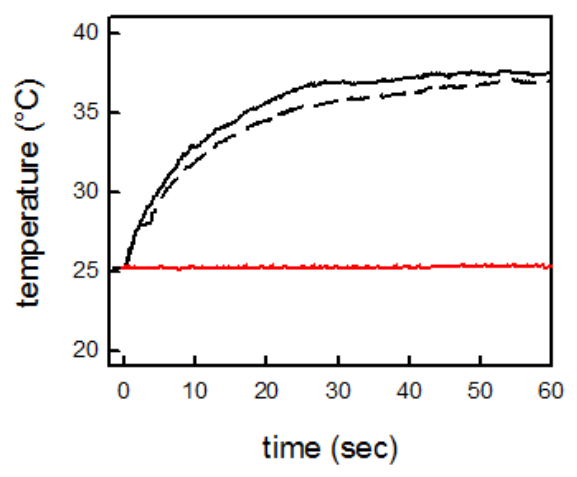

Figure 3. Temperature increase as a function of irradiation time, measured under NIR laser irradiation $\left(808 \mathrm{~nm}, 0.26 \mathrm{~W} / \mathrm{cm}^{2}\right)$ for a freshly prepared GLASS-PEI-TRI sample (black solid line) and for a GLASS-PEI-TRI sample after an immersion of $48 \mathrm{~h}$ in water (black dashed line). The red solid line represents the thermogram obtained for an uncoated glass in the same conditions.

\subsection{Antibacterial Features of GLASS-PEI-TRI Samples}

As a first step to evaluate the antibacterial features, we investigated the microbicidal effect (ME) of GLASS-PEI-TRI samples against E. coli and S. aureus, which are representative strains of Gram- and Gram + bacteria, respectively. We have recently introduced a procedure which allows the evaluation of the ME of a properly coated surface in contact with a thin liquid film containing bacteria, using the following formula:

$$
\mathrm{ME}=\log N_{\mathrm{C}}-\log N_{\mathrm{E}}
$$


where $N_{\mathrm{C}}$ is the number of $\mathrm{CFU} / \mathrm{mL}$ developed on the unmodified control glass, and $N_{\mathrm{E}}$ is the number of $\mathrm{CFU} / \mathrm{mL}$ counted after exposure to modified glass ( $\mathrm{CFU}=$ colony forming unit).

We chose three contact times ( $15 \mathrm{~min}, 5 \mathrm{~h}$, and $24 \mathrm{~h}$ ) and evaluated the ME after these intervals, which are reported in Table 1 . The longer contact time was set to $24 \mathrm{~h}$, as biofilm colonies usually develop on biomaterials in a $12-18 \mathrm{~h}$ period from the bacterial contamination: thus, an antibacterial activity lasting for $24 \mathrm{~h}$ is an optimal feature to impart the ability to prevent biofilm growth to biomedical surfaces [36]. Results show that GLASS-PEI-TRI glass slides exert a significant bactericidal effect that increases with the contact time. For $E$. coli, a contact time of $5 \mathrm{~h}$ is enough to eliminate at least $99.9 \%$ of bacteria cells. After $24 \mathrm{~h}$, more than $99 \%$ of S. aureus are killed too. It is important to notice that tests with contact times longer than $24 \mathrm{~h}$ suffer from a main limitation: in the conditions of the experiment, in the timescale of days, the bacteria in the control samples also tend to die, thus reducing the number of colony forming units and giving poorly reproducible and reliable values of ME. Anyway, preliminary tests performed with a $48 \mathrm{~h}$ contact time showed that ME does not increase when the contact time is prolonged for more than $24 \mathrm{~h}$. The effect on $\mathrm{S}$. aureus seems a little less intense with $5 \mathrm{~h}$ of contact, while no microbicidal effect towards either strain is evident within the short (15 min) contact time. This is consistent with precedent studies on AgNP-coated antibacterial surfaces: as we have reported for similar systems [34,36,37], one should expect a very limited release of silver ions for short (less than $1 \mathrm{~h}$ ) contact times, and this explains the low ME observed in these timescales. Mechanisms by which AgNPs exert their antibacterial effect is still debated; however, it was recently shown [38] that silver nano-objects are devoid of antibacterial action when used under anaerobic conditions, which exclude oxidation to silver (I), thus preventing its release. This is consistent with our proposal [34] of the formation of an oxidized silver layer on the surface of citrate-coated grafted nano-objects, reaching a steady-state in which $\mathrm{Ag}^{+}$ions are slowly released, continuously being replaced by $\mathrm{Ag}$ oxidized from the bulk of the nano-objects. Remarkably, in the tests proposed by CEN (European Committee for Standardization) in EN 13697, the microbicidal activity of a disinfectant is considered acceptable when the decimal-log reduction rate (which is strictly related to our ME) is at least equal to 4 after 5 min of contact. In our case the ME of the modified glass is very close to 4 after $24 \mathrm{~h}$ of contact with E. coli, showing a long-term action that seems well suited for building antibacterial surfaces for medical devices.

Table 1. Microbicidal effect (ME) values ${ }^{1}$ for GLASS-PEI-TRI samples.

\begin{tabular}{cccc}
\hline Bacterial Strain & $\mathbf{1 5} \mathbf{~} \mathbf{m i n}$ & $\mathbf{5 ~ h}$ & $\mathbf{2 4} \mathbf{~ h}$ \\
\hline E. coli & $<0.3$ & $3.2(0.3)$ & $3.6(0.3)$ \\
S. aureus & $<0.3$ & $0.9(0.3)$ & $2.5(0.3)$ \\
\hline are obtained as the average of three measurements. ME $=\log N_{\mathrm{C}}$ & $\log N_{\mathrm{E}}$.
\end{tabular}

This antibacterial effect, anyway, is not a surprise: it was largely expected and substantially confirms the behavior obtained with spherical nanoparticles. We were much more interested in the new features that are introduced by the anisotropic shape on nanoplates (i.e., the possibility to efficiently absorb NIR laser radiation, to dissipate it as heat, and to exploit this hyperthermia to kill bacteria cells). In literature, no precise information is present concerning the temperature values needed to eliminate bacteria with hyperthermia caused by a photo-thermal effect. It is reported that the heating of E. coli cells at temperatures of $52{ }^{\circ} \mathrm{C}$ for a time longer than 5 min causes the destruction of the permeability barrier of bacteria cells [39]. It is also reported that laser irradiation of a cluster of gold nanorods for $5 \mathrm{~min}$ allows a temperature of about $60^{\circ} \mathrm{C}$ to be reached, thus destroying most bacterial cells in a biofilm of E. coli [18]. Recently it was described how NIR laser irradiation of a monolayer of GNSs on a glass bulk surface for 30 min causes a few degrees hyperthermia, which is enough to almost completely destroy a bacteria colony in a S. aureus biofilm layer [20]. In any case, we must stress the fact that "macroscopic" effects, especially when described by thermograms registered with a thermocamera, only show temperature changes of relatively large areas (in the range 
of $1 \mathrm{~cm}^{2}$ ) of bulk glass samples, changes which are, of course, the function of several experimental parameters [19]. So, information on the temperatures that are reached in the close surroundings of the nano-objects' surfaces are completely missed, even if one can reasonably state that these temperatures are considerably higher than those measured by thermograms. From the reported literature data, anyway, it is possible to speculate that also a few degrees hyperthermia measured on a bulk surface could be useful to produce a strong effect on bacteria health [28].

Thus, we moved toward the investigation of the antibacterial action obtained by laser-induced photo-thermal effects causing localized hyperthermia. GLASS-PEI-TRI samples-reduced to appropriate dimensions in order to be completely covered by the laser spot-were irradiated for $15 \mathrm{~min}$ with an $808 \mathrm{~nm}$ laser source, while in contact with the described bacteria suspensions, using an irradiance of $0.26 \mathrm{~W} / \mathrm{cm}^{2}$. In this case, the "thermal microbicidal effect", $\mathrm{ME}_{\mathrm{T}}$, was calculated with the following formula:

$$
\mathrm{ME}_{\mathrm{T}}=\log N_{\mathrm{C}}-\log N_{\mathrm{T}}
$$

where $N_{C}$ is the number of $\mathrm{CFU} / \mathrm{mL}$ developed after the $15 \mathrm{~min}$ contact with GLASS-PEI-TRI samples in absence of irradiation, taken as control, and $N_{\mathrm{T}}$ is the number of CFU/mL counted after $15 \mathrm{~min}$ of contact with GLASS-PEI-TRI samples in presence of NIR laser irradiation. It is important to stress that, in these conditions, $\mathrm{ME}_{\mathrm{T}}$ must be ascribed only to the photo-thermal effect, as any possible influence from the (limited) silver release occurring in absence of irradiation is already considered in the control count. Moreover, we have the evidence that no silver-related ME is observed during the first $15 \mathrm{~min}$ of contact, as already reported in Table 1. It is also important to stress that when the experiment is repeated using nonfunctionalized glass samples, the $\mathrm{ME}_{\mathrm{T}}$ is absent $(<0.3)$. This clearly indicates that laser irradiation alone, in absence of plasmonic objects exerting photo-thermal features, does not cause any harm to bacteria. The same set of experiments were replicated on a set of samples after their immersion in water for $24 \mathrm{~h}$, showing the same $\mathrm{ME}_{\mathrm{T}}$ values, thus confirming stability of the sample features after long immersions times in water.

The $\mathrm{ME}_{\mathrm{T}}$ test results show that the laser treatment kills more than $99.7 \%$ of E. coli and about $97 \%$ of $S$. aureus cells in $15 \mathrm{~min}$ of irradiation at $0.26 \mathrm{~W} / \mathrm{cm}^{2}$, a value which is below the maximum allowed for exposure of the skin [40], and using a wavelength which is suitable for in vivo use. As reported in Table 2, in the case of $\mathrm{ME}_{\mathrm{T}}$, we observe a higher activity against $E$. coli than against $S$. aureus, which was somewhat already observed in the values of ME that were due only to silver release. This may be due to the fact that Gram-positive bacteria present a relatively thick $(20-80 \mathrm{~nm})$ and continuous cell wall consisting mainly of peptidoglycan, while Gram-negative bacteria feature a thinner peptidoglycan layer $(5-10 \mathrm{~nm})$ surrounded by an outer phospholipidic membrane, which is expected to be less solid and less resistant $[9,10,28]$. In this case, hyperthermia caused by the photo-thermal effect seems particularly efficient in killing Gram-negative bacteria. As immersion in water for $24 \mathrm{~h}$ does not change the shape, dimensions, LSPR features, or the photo-thermal behavior of objects, it obviously has no effect on hyperthermia and subsequently induced antibacterial activity, which is conserved. This behavior allows one to imagine the use of the laser switched, hyperthermia-related, thermal ME after the surface has already exerted its silver release-related microbicidal action (described by ME values reported in Table 1), in all the cases in which an "extra" action is needed.

Table 2. Thermal ME $\left(\mathrm{ME}_{\mathrm{T}}\right)$ values ${ }^{1}$ for GLASS-PEI-TRI samples.

\begin{tabular}{ccc}
\hline Bacterial Strain & $\mathbf{1 5} \mathbf{~ m i n}$ & $\mathbf{1 5} \mathbf{~ m i n}$ (after $\mathbf{2 4} \mathbf{h}$ in Water) \\
\hline E. coli & $2.5(0.3)$ & $2.4(0.3)$ \\
S. aureus & $1.5(0.3)$ & $1.5(0.3)$ \\
\hline Values are obtained as the average of three measurements. $\mathrm{ME}_{\mathrm{T}}=\log N_{\mathrm{C}}-\log N_{\mathrm{T}}$
\end{tabular}




\section{Materials and Methods}

\subsection{Materials}

Silver nitrate $(>99.8 \%)$, sodium borohydride $(>99.0 \%)$, sodium citrate (TSC) $(>99.0 \%)$, and ascorbic acid $\geq 99 \%$ were purchased from Sigma-Aldrich (St. Louis, MO, USA)). Trimethoxysilylpropyl (polyethylenimine) (50\% in isopropanol) was purchased from Gelest Inc. (Morrisville, PA, USA). Reagents were used as received. Solvents were purchased from Sigma-Aldrich (St. Louis, MO, USA)) and used as supplied. Microscopy cover glass slides $(21 \times 26 \mathrm{~mm})$ were purchased from DelChimica (Milano, Italy). Water was deionized and then bi-distilled.

\subsection{Seeds Preparation}

Seeds, spherical silver nanoparticles, were prepared according to a reported method [35]. To $20.0 \mathrm{~mL}$ of distilled water, the following solutions were added in sequence under vigorous stirring: $0.100 \mathrm{~mL}$ of a solution of $\mathrm{AgNO}_{3} 0.018 \mathrm{M}, 0.100 \mathrm{~mL}$ of a solution of TSC $0.017 \mathrm{M}$, and $0.600 \mathrm{~mL}$ of a solution of $\mathrm{NaBH}_{4} 0.010 \mathrm{M}$. After the last addition, stirring was immediately stopped in order to avoid coagulation. The colloidal suspensions were stored in the preparation flask and maintained in the dark. Then, this colloidal suspension of seeds was diluted with distilled water to reach an absorbance value of 0.5 units to obtain a stock solution, which was used for preparation of silver nanoplates.

\subsection{Synthesis of Silver Nanoplates}

Silver nanoplates were synthesized by a seed-mediated growth method. Firstly, growth solution was prepared in a beaker under magnetic stirring, adding $20.0 \mathrm{~mL}$ of a solution of TSC $8.5 \times 10^{-3} \mathrm{M}$, $12 \mathrm{~mL}$ of a solution of $\mathrm{AgNO}_{3} 5 \times 10^{-4} \mathrm{M}$, and $0.300 \mathrm{~mL}$ of ascorbic acid $1 \times 10^{-2} \mathrm{M}$. Secondly, $0.300 \mathrm{~mL}$ of the stock solution of seeds was added and magnetic stirring was instantly stopped. A changing color of solution could be observed from pale yellow to dark pink, and finally to a blue/grey color, after approximately $12 \mathrm{~h}$. Silver nanoplates colloids were stored at $25{ }^{\circ} \mathrm{C}$ and used without further treatments.

\subsection{Preparation of PEI-Silane Self-Assembled Monolayers on Glass Surface (SURF-PEI Glass)}

The samples were prepared according a reported method [28]. Briefly: glass substrates were cleaned for $30 \mathrm{~min}$ in freshly prepared piranha solution (3:1 v/v $\mathrm{H}_{2} \mathrm{SO}_{4}: \mathrm{H}_{2} \mathrm{O}_{2}(30 \%)$ (Caution! Piranha solution is a strong oxidizing agent and should be handled with care). Glass substrates were then washed three times with ultrapure water in a sonic bath and oven-dried. The slides were then immersed for $6 \mathrm{~min}$ in a $4 \%(v / v)$ solution of PEI-silane in ethanol at room temperature. In a typical preparation, eight glass slides were prepared at the same time (i.e., reacting in the same silane solution inside an eight-place holder (a microscope glass slides staining jar)). After this, the slides were washed two times with ethanol and one time with ultrapure water in a sonic bath and blow-dried with nitrogen.

\subsection{Preparation of GLASS-PEI-TRI}

The silver nanoplates were grafted on SURF-PEI modified glass slides. In a typical preparation, eight glass slides were covered with a colloidal suspension of silver nanoplates inside an eight-place holder (a microscope glass slide staining jar) for $14 \mathrm{~h}$ at $25^{\circ} \mathrm{C}$. Finally, functionalized glass slides were rinsed three times with distilled water and gently dried under nitrogen flux.

\subsection{Antibacterial Activity Tests}

The antibacterial activity of functionalized cover glasses was investigated against Staphylococcus aureus ATCC 6538 (Gram+) and Escherichia coli ATCC 10356 (Gram-). The microorganisms were grown overnight in Tryptone Soya Broth (Oxoid; Basingstoke, Hampshire, UK) at $37^{\circ} \mathrm{C}$. Washed cells were resuspended in Dulbecco's phosphate-buffered saline (PBS) and optical density (OD) was adjusted to 
0.2 at $650 \mathrm{~nm}$ wavelength, corresponding approximately to $1 \times 10^{8} \mathrm{CFU} / \mathrm{mL}$. Bacterial suspension $(10 \mu \mathrm{L})$ was deposited onto a standard glass slide $(76 \times 26 \mathrm{~mm})$, then the microbial suspension was covered with a functionalized cover glass slide $(21 \times 26 \mathrm{~mm})$, forming a thin film between the slides that facilitates direct contact of the microorganisms with the active surface. The two assembled glasses were introduced in a Falcon test tube $(50 \mathrm{~mL})$ containing $1 \mathrm{~mL}$ of PBS to maintain a damp environment. In this test, microbes are incubated in non-nutritive suspensions that do not give the microorganisms the potential to grow during the test. For each bacterial strain, three equivalent modified glass slides were prepared; the slides were maintained in contact with the liquid films containing bacteria at room temperature for $15 \mathrm{~min}, 5 \mathrm{~h}$, and $24 \mathrm{~h}$, respectively; for each time of contact, an unmodified glass slide was treated in the same way as control sample. After the specified times of contact had elapsed, $9 \mathrm{~mL}$ of PBS was introduced in each Falcon test tube under gentle shaking to detach the assembled glass slides. Bacterial suspensions were then grown in Tryptone Soya Agar (Oxoid; Basingstoke, Hampshire, UK) to count viable cells. The decimal-log reduction rate (i.e., the microbicidal effect (ME)) was calculated using the formula:

$$
\mathrm{ME}=\log N_{\mathrm{C}}-\log N_{\mathrm{E}}
$$

( $N_{\mathrm{C}}$ being the number of $\mathrm{CFU} / \mathrm{mL}$ developed on the unmodified control glasses, and $N_{\mathrm{E}}$ being the number of $\mathrm{CFU} / \mathrm{mL}$ counted after exposure to modified glasses). The results expressed as $\mathrm{ME}$ represent the average of three equivalent determinations. This method can be considered as a version of the Japanese Industrial Standard JIS Z 2811 developed to measure the antibacterial activity of plastic surfaces.

\subsection{Thermal Microbicidal Tests}

Antibacterial activity due to the photo-thermal effect of was investigated against Staphylococcus aureus ATCC 6538 (Gram+) and Escherichia coli ATCC 10356 (Gram-). One functionalized slide was cut in four sections of $10 \times 10 \mathrm{~mm}$ in order to be completely irradiated by laser. A volume of $0.020 \mathrm{~mL}$ of bacterial suspension was deposited on two sections. For each pair of functionalized glass slides, one was irradiated for $15 \mathrm{~min}$, whereas the other was not irradiated. After this time, the glass section covered with bacterial suspensions was suspended in $1 \mathrm{~mL}$ of sterile water, then it was taken away and water was used to make three dilutions in 3 different tubes, in which $5 \mathrm{~mL}$ of sterile water was contained: 1:100, 1:10,000, 1:100,000. From each tube, $1 \mathrm{~mL}$ of suspension was taken and then grown in Tryptone Soya Agar (Oxoid; Basingstoke, Hampshire, UK) to count viable cells. The decimal-log reduction rate (i.e., the "thermal microbicidal effect") $\mathrm{ME}_{\mathrm{T}}$ was calculated with the following formula

$$
\mathrm{ME}_{\mathrm{T}}=\log N_{\mathrm{C}}-\log N_{\mathrm{T}}
$$

where $N_{C}$ is the number of $\mathrm{CFU} / \mathrm{mL}$ developed in contact with a nonirradiated modified control glass sample, and $N_{\mathrm{T}}$ the number of $\mathrm{CFU} / \mathrm{mL}$ counted after exposure to modified glass samples and irradiation. Each experiment was repeated three times.

\subsection{Instrumentation and Instrumental Methods}

Absorbance spectra of colloidal suspensions were taken with a Varian Cary 100 spectrophotometer (Agilent, Santa Clara, CA, USA) in the 200-1100 nm range, and spectra of functionalized glass slides were obtained placing the slides on the Varian Cary 100 spectrophotometer equipped with a dedicated Varian solid sample holder.

TEM images were obtained on colloidal solutions of silver nanoplates diluted five times with distilled water, deposited on Nickel grids (300 mesh), covered with a Parlodion membrane, and observed with a Jeol JEM-1200 EX II instrument (Jeol Ltd., Tokyo, Japan).

Zeta potential was measured with a Zetasizer Nano ZS90 Malvern instrument Malvern Instruments Ltd., Malvern, UK), equipped with dedicated cuvettes. Measurements were repeated three times using $1 \mathrm{~mL}$ of colloidal suspension for each one. 
SEM images were taken from Tescan Mira XMU variable pressure field emission gun scanning electron microscope-FEG SEM (Tescan USA Inc., Warrendale, PA, USA). Slides were mounted onto aluminum stubs using double-sided carbon adhesive tape and were then made electrically conductive by coating in vacuum with a thin layer of $\mathrm{Pt} / \mathrm{Pd}(2.5 \mathrm{~nm})$. Observations were made in backscattered electrons mode (BSE) at $30 \mathrm{kV}$ and with InBeam secondary electron detector for higher spatial resolution.

Thermograms (temperature vs. time) were recorded on SURF-PEI-TRI samples by means of a laser (multimode AlGaAs laser diode, L808P200, Thorlabs GmbH (Thorlabs GmbH Dachau/Munich, Germany), emitting light at the wavelength of about $808 \mathrm{~nm}$, power of radiation is $200 \mathrm{~mW}$, irradiance is $0.26 \mathrm{~W} / \mathrm{cm}^{2}$ ). Temperature was recorded by means of a FLIR E40 thermocamera and FLIR Tools + software (FLIR systems, Wilsonville, OR, USA).

The total Ag content of GLASS-PEI-TRI was determined by quantitatively oxidizing the silver plates grafted on a single slide by dipping it in $3 \mathrm{~mL}$ ultrapure concentrated $\mathrm{HNO}_{3}$ diluted 1:5 with water (13\% $w / v$ as final concentration) in a beaker, and keeping it overnight at RT on a Heidolph Promax 1020 (Heidolph Instruments GmbH \& Co. KG, Schwabach, Germany) reciprocating platform shaker. The Ag content in solution was then determined by inductively coupled plasma optical emission spectroscopy (ICP-OES) with an ICP-OES OPTIMA 3000 PerkinElmer instrument (Perkin Elmer Inc., Waltham, MA, USA). The measure was repeated on eight glass samples coming from four different preparations.

Release of $\mathrm{Ag}^{+}$in water was measured on a set four 4 functionalized slides $(21 \times 26 \mathrm{~mm}$ coated on both sides, total coated surface $10.92 \mathrm{~cm}^{2}$ ) prepared as described above. Each slide was then immersed in $3 \mathrm{~mL}$ of ultrapure water. Slides were taken off the water after $48 \mathrm{~h}$. A UV-vis spectra was measured on the water solution, and then the content of $\mathrm{Ag}^{+}$was determined by ICP-OES. Measurements were repeated three times, and mean values are given. ICP data were collected with an ICP-OES OPTIMA 3000 PerkinElmer instrument (Perkin Elmer Inc., Waltham, MA, USA). UV-vis spectra were collected on the glass samples after the immersion. One glass sample was then analyzed by SEM (Tescan USA Inc., Warrendale, PA USA).

To prepare a sample of nanoplates synthesized with standard preparation for XRD measurement, $10 \mathrm{~mL}$ of colloidal suspension was centrifuged for $20 \mathrm{~min}$ at $5000 \mathrm{rpm}$ and resuspended in $3 \mathrm{~mL}$ of bi-distilled water. This volume was centrifuged again, resuspended in a drop of water, and finally dropped on a blank glass slide and dried for one night. XRD of GLASS-PEI-TRI samples were measured directly on a functionalized slide obtained as described in Section 3.5. XRD measurements were performed by using a Bruker D5005 diffractometer (Bruker, Billerica, MA, USA) with the Cu K $\alpha$ radiation, graphite monochromator and scintillation detector. The patterns were collected with a step size of $0.03^{\circ}$ and counting time of $2 \mathrm{~s}$ per step in the angular range $20^{\circ}-65^{\circ}$.

\section{Conclusions}

The GLASS-PEI-TRI samples exert an antibacterial action, which seems particularly active for Gram- bacteria like E. coli, exploiting two separate mechanisms. One is the long-term action given by the "classical" silver ion release, which can be used to prevent the formation and adhesion of colonies over a long period of time, while the other is the laser-switchable photo-thermal action, which can be activated based on need (for example, in the case of infections not sufficiently prevented by the first mechanism) and obtained using the peculiar spectral features of the objects grafted on the surface, objects which can be tailored in order to have their LSPR spectra matching with the wavelength of the desired laser source.

In our case, the laser-switched antibacterial activity is obtained by irradiation of bulk surfaces in the NIR (which could be used in vivo) using a radiation intensity which does not damage the tissues. LSPR absorption was placed close to the laser wavelength at $808 \mathrm{~nm}$, using a simple synthesis which avoids reactants that can be dangerous to human health. The typical antibacterial features usually observed in surfaces coated with silver nano-objects—-which are due essentially to the controlled release 
of $\mathrm{Ag}^{+}$ions, and which is ensured by the verified stability of the grafted nano-objects layers-are conserved. As glasslike $\mathrm{SiO}_{2}$ films can be easily deposited on a large spectrum of materials by gelification of a siloxane sol, the procedures herein described-involving a first silanization step of a glass sample — can also be applied to coat a wide range of materials. Thus, these results could be useful for applications to several types of materials of medical use (prosthetic and subcutaneous devices, surgical sutures, wound dressings, to name a few examples). All these kinds of bulk materials surely need long-term antibacterial surface protection, here ensured by the ability of silver nano-objects to release small and controlled amounts of silver ions for long contact times (some days), but will also profit by a switchable, very quick (a few minutes) laser treatment to ensure an "extra" elimination of bacteria cells, when needed.

Supplementary Materials: The following are available online at http://www.mdpi.com/2079-4991/7/1/7/s1, Figure S1: TEM image of seeds preparation, Figure S2: UV-vis spectra of different stock solutions of seeds, Figure S3: UV-vis spectra showing the kinetic of evolution with time of a typical seed-growth preparation of silver nanoplates, Figure S4: UV-vis spectra of different colloidal suspensions of silver nanoplates obtained with the standard preparation, Figure S5-S7: TEM images of objects obtained for standard nanoplates preparations, Figure S8: Histogram representing the distribution of the average altitude values measured for 43 regular triangles obtained from a standard preparation. Figure S9: TEM images of nanoplates obtained decreasing of one third the seed quantity (in respect to the standard preparation). Figure S10: TEM images of nanoplates obtained doubling the seed quantity (in respect to the standard preparation), Figure S11: UV-vis spectra of four GLASS-PEI-TRI samples obtained in four different reaction vessels, coming from four identical preparations. Figure S12: UV-vis spectra of seven GLASS-PEI-TRI samples obtained in the same reaction vessel during a single preparation. Figure S13: UV-vis spectra of the same GLASS-PEI-TRI sample before (solid line) and after (dashed line) immersion in water for $48 \mathrm{~h}$, Figure S14: SEM images of sample of GLASS-PEI-TRI after $48 \mathrm{~h}$ of immersion in water: (a) large area image; $(b-d)$ images of triangular objects, which retains morphology and dimension observed in the fresh samples, Figure S15: XRD data.

Acknowledgments: We acknowledge the support of University of Pavia (Fondo Ricerca Giovani 2015). We also gratefully acknowledge M.P. Riccardi, Arvedi Laboratory, CISRiC, Pavia, for carrying out SEM experiments. Authors also want to thank Vittorio Necchi and Centro Grandi Strumenti, Pavia, for TEM images

Author Contributions: A.T. and P.P. conceived and designed the experiments; A.D. and R.D. made all the synthesis and performed some characterization experiments; P.G. conceived and performed the microbicidal measurements, G.D. performed the experiments involving laser irradiation, L.C., M.P. and M.B. made the most of the characterizations of the bulk samples; A.T. wrote the paper.

Conflicts of Interest: The authors declare no conflict of interest.

\section{References}

1. Johnstone, T.C.; Suntharalingam, K.; Lippard, S.J. The Next Generation of Platinum Drugs: Targeted Pt(II) Agents, Nanoparticle Delivery, and Pt(IV) Prodrugs. Chem. Rev. 2016, 116, 3436-3486. [CrossRef] [PubMed]

2. Yamamoto, E.; Kazuyuki, K. Colloidal Mesoporous Silica Nanoparticles. Bull. Chem. Soc. Jpn. 2016, 89, 501-539. [CrossRef]

3. Li, Z.; Hao, H.; Tang, S.; Li, Y.; Yu, X.F.; Wang, H.; Li, P.; Sun, Z.; Zhang, H.; Liu, C.; et al. Small gold nanorods laden macrophages for enhanced tumor coverage in photothermal therapy. Biomaterials 2016, 74, 144-154. [CrossRef] [PubMed]

4. Abe, H.; Liu, J.; Ariga, K. Catalytic nanoarchitectonics for environmentally compatible energy generation. Mater. Today 2016, 19, 12-18. [CrossRef]

5. McGann, P.; Snesrud, E.; Maybank, R.; Corey, B.; Ong, A.C.; Clifford, R.; Hinkle, M.; Whitman, T.; Lesho, E.; Schaecher, K.E. Escherichia coli Harboring Mcr-1 and Bla(CTX-M) on a Novel IncF Plasmid: First Report of Mcr-1 in the United States. Antimicrob. Agents Chemother. 2016, 60, 4420-4421. [CrossRef] [PubMed]

6. Campoccia, D.; Montanaro, L.; Arciola, C.R. A review of the clinical implications of anti-infective biomaterials and infection-resistant surfaces. Biomaterials 2013, 34, 8018-8029. [CrossRef] [PubMed]

7. Pallavicini, P.; Dacarro, G.; Diaz-Fernandez, Y.A.; Taglietti, A. Coordination chemistry of surface-grafted ligands for antibacterial materials. Coord. Chem. Rev. 2014, 275, 37-53. [CrossRef]

8. Morones, J.R.; Elechiguerra, J.L.; Camacho, A.; Holt, K.; Kouri, J.B.; Ramirez, J.T.; Yacaman, M.J. The bactericidal effect of silver nanoparticles. Nanotechnology 2005, 16, 2346-2353. [CrossRef] [PubMed] 
9. Taglietti, A.; Fernandez, Y.A.D.; Amato, E.; Cucca, L.; Dacarro, G.; Grisoli, P.; Necchi, V.; Pallavicini, P.; Pasotti, L.; Patrini, M. Antibacterial Activity of Glutathione-Coated Silver Nanoparticles against Gram Positive and Gram Negative Bacteria. Langmuir 2012, 28, 8140-8148. [CrossRef] [PubMed]

10. Amato, E.; Diaz-Fernandez, Y.A.; Taglietti, A.; Pallavicini, P.; Pasotti, L.; Cucca, L.; Milanese, C.; Grisoli, P.; Dacarro, C.; Fernandez-Hechavarria, J.M.; et al. Synthesis, Characterization and Antibacterial Activity against Gram Positive and Gram Negative Bacteria of Biomimetically Coated Silver Nanoparticles. Langmuir 2011, 27, 9165-9173. [CrossRef] [PubMed]

11. Pal, S.; Tak, Y.K.; Song, J.M. Does the Antibacterial Activity of Silver Nanoparticles Depend on the Shape of the Nanoparticle? A Study of the Gram-Negative Bacterium Escherichia coli. Appl. Environ. Microbiol. 2007, 73, 1712-1720. [CrossRef] [PubMed]

12. Tang, B.; Li, J.L.; Hou, X.L.; Afrin, T.; Sun, L.; Wang, X.G. Colorful and Antibacterial Silk Fiber from Anisotropic Silver Nanoparticles. Ind. Eng. Chem. Res. 2013, 52, 4556-4563. [CrossRef]

13. Vukoje, I.; Lazic, V.; Vodnik, V.; Mitric, M.; Jokic, B.; Ahrenkiel, S.P.; Nedeljkovic, J.M.; Radetic, M. The influence of triangular silver nanoplates on antimicrobial activity and color of cotton fabrics pretreated with chitosan. J. Mater. Sci. 2014, 49, 4453-4460. [CrossRef]

14. Gao, M.J.; Sun, L.; Wang, Z.Q.; Zhao, Y.B. Controlled synthesis of Ag nanoparticles with different morphologies and their antibacterial properties. Mater. Sci. Eng. C 2013, 33, 397-404. [CrossRef] [PubMed]

15. El-Zahry, M.R.; Mahmoud, A.; Refaat, I.H.; Mohamed, H.A.; Bohlmann, H.; Lendl, B. Antibacterial effect of various shapes of silver nanoparticles monitored by SERS. Talanta 2015, 138, 183-189. [CrossRef] [PubMed]

16. Alarcon, E.I.; Vulesevic, B.; Argawal, A.; Ross, A.; Bejjani, P.; Podrebarac, J.; Ravichandran, R.; Phopase, J.; Suuronen, E.J.; Griffith, M. Coloured cornea replacements with anti-infective properties: Expanding the safe use of silver nanoparticles in regenerative medicine. Nanoscale 2016, 8, 6484-6489. [CrossRef] [PubMed]

17. De Aberasturi, D.J.; Serrano-Montes, A.B.; Liz-Marzan, L.M. Modern Applications of Plasmonic Nanoparticles: From Energy to Health. Adv. Opt. Mater. 2015, 3, 602-617. [CrossRef]

18. Jo, W.; Kim, M.J. Influence of the photothermal effect of a gold nanorod cluster on biofilm disinfection. Nanotechnology 2013, 24, 195104. [CrossRef] [PubMed]

19. Khan, S.A.; Singh, A.K.; Senapati, D.; Fan, Z.; Ray, P.C. Bio-conjugated popcorn shaped gold nanoparticles for targeted photothermal killing of multiple drug resistant Salmonella DT104. J. Mater. Chem. 2011, 21, 17705-17709. [CrossRef]

20. Pallavicini, P.; Dona, A.; Taglietti, A.; Minzioni, P.; Patrini, M.; Dacarro, G.; Chirico, G.; Sironi, L.; Bloise, N.; Visai, L.; et al. Self-assembled monolayers of gold nanostars: A convenient tool for near-IR photothermal biofilm eradication. Chem. Commun. 2014, 50, 1969-1971. [CrossRef] [PubMed]

21. Boca, S.C.; Potara, M.; Gabudean, A.M.; Juhem, A.; Baldeck, P.L.; Astilean, S. Chitosan-coated triangular silver nanoparticles as a novel class of biocompatible, highly effective photothermal transducers for in vitro cancer cell therapy. Cancer Lett. 2011, 311, 131-140. [CrossRef] [PubMed]

22. Boca-Farcau, S.; Potara, M.; Simon, T.; Juhem, A.; Baldeck, P.; Astilean, S. Folic Acid-Conjugated, SERS-Labeled Silver Nanotriangles for Multimodal Detection and Targeted Photothermal Treatment on Human Ovarian Cancer Cells. Mol. Pharm. 2014, 11, 391-399. [CrossRef] [PubMed]

23. Pastoriza-Santos, I.; Liz-Marzan, L.M. Colloidal silver nanoplates. State of the art and future challenges. J. Mater. Chem. 2008, 18, 1724-1737. [CrossRef]

24. Aherne, D.; Ledwith, D.M.; Gara, M.; Kelly, J.M. Optical properties and growth aspects of silver nanoprisms produced by a highly reproducible and rapid synthesis at room temperature. Adv. Funct. Mater. 2008, 18, 2005-2016. [CrossRef]

25. Mo, S.; Chen, X.; Chen, M.; He, C.; Lu, Y.; Zheng, N. Two-dimensional antibacterial Pd@Ag nanosheets with a synergetic effect of plasmonic heating and $\mathrm{Ag}^{+}$release. J. Mater. Chem. B 2015, 3, 6255-6260. [CrossRef]

26. Fasciani, C.; Jazmin Silvero, M.; Alexandra Anghel, M.; Argueello, G.A.; Cecilia Becerra, M.; Scaiano, J.C. Aspartame-Stabilized Gold-Silver Bimetallic Biocompatible Nanostructures with Plasmonic Photothermal Properties, Antibacterial Activity, and Long-Term Stability. J. Am. Chem. Soc. 2014, 136, 17394-17397. [CrossRef] [PubMed]

27. Black, K.C.L.; Sileika, T.S.; Yi, J.; Zhang, R.; Rivera, J.G.; Messersmith, P.B. Bacterial Killing by Light-Triggered Release of Silver from Biomimetic Metal Nanorods. Small 2014, 10, 169-178. [CrossRef] [PubMed] 
28. D'Agostino, A.; Taglietti, A.; Grisoli, P.; Dacarro, G.; Cucca, L.; Patrini, M.; Pallavicini, P. Seed mediated growth of silver nanoplates on glass: Exploiting the bimodal antibacterial effect by near IR photo-thermal action and $\mathrm{Ag}^{+}$release. RSC Adv. 2016, 6, 70414-70423.

29. Casu, A.; Cabrini, E.; Dona, A.; Falqui, A.; Diaz-Fernandez, Y.; Milanese, C.; Taglietti, A.; Pallavicini, P. Controlled Synthesis of Gold Nanostars by Using a Zwitterionic Surfactant. Chem. Eur. J. 2012, 18, 9381-9390. [CrossRef] [PubMed]

30. Mahmoud, M.A.; Tabor, C.E.; El-Sayed, M.A.; Ding, Y.; Wang, Z.L. A new catalytically active colloidal platinum nanocatalyst: The multiarmed nanostar single crystal. J. Am. Chem. Soc. 2008, 130, 4590. [CrossRef] [PubMed]

31. Sun, Y.G. Direct growth of dense, pristine metal nanoplates with well-controlled dimensions on semiconductor substrates. Chem. Mater. 2007, 19, 5845-5847. [CrossRef]

32. Alkilany, A.M.; Nagaria, P.K.; Hexel, C.R.; Shaw, T.J.; Murphy, C.J.; Wyatt, M.D. Cellular Uptake and Cytotoxicity of Gold Nanorods: Molecular Origin of Cytotoxicity and Surface Effects. Small 2009, 5, 701-708. [CrossRef] [PubMed]

33. Ledwith, D.M.; Whelan, A.M.; Kelly, J.M. A rapid, straight-forward method for controlling the morphology of stable silver nanoparticles. J. Mater. Chem. 2007, 17, 2459-2464. [CrossRef]

34. Pallavicini, P.; Taglietti, A.; Dacarro, G.; Diaz-Fernandez, Y.A.; Galli, M.; Grisoli, P.; Patrini, M.; De Magistris, G.S.; Zanoni, R. Self-assembled monolayers of silver nanoparticles firmly grafted on glass surfaces: Low $\mathrm{Ag}^{+}$release for an efficient antibacterial activity. J. Colloid Interface Sci. 2010, 350, 110-116. [CrossRef] [PubMed]

35. Sung, H.K.; Oh, S.Y.; Park, C.; Kim, Y. Colorimetric detection of $\mathrm{Co}^{2+}$ ion using silver nanoparticles with spherical, plate, and rod shapes. Langmuir 2013, 29, 8978-8982. [CrossRef] [PubMed]

36. Taglietti, A.; Arciola, C.R.; D’Agostino, A.; Dacarro, G.; Montanaro, L.; Campoccia, D.; Cucca, L.; Vercellino, M.; Poggi, A.; Pallavicini, P. Antibiofilm activity of a monolayer of silver nanoparticles anchored to an amino-silanized glass surface. Biomaterials 2014, 35, 1779-1788. [CrossRef] [PubMed]

37. Dacarro, G.; Cucca, L.; Grisoli, P.; Pallavicini, P.; Patrini, M.; Taglietti, A. Monolayers of polyethilenimine on flat glass: A versatile platform for cations coordination and nanoparticles grafting in the preparation of antibacterial surfaces. Dalton Trans. 2012, 41, 2456-2463. [CrossRef] [PubMed]

38. Xiu, Z.M.; Zhang, Q.B.; Puppala, H.L.; Colvin, V.L.; Alvarez, P.J.J. Negligible Particle-Specific Antibacterial Activity of Silver Nanoparticles. Nano Lett. 2012, 12, 4271-4275. [CrossRef] [PubMed]

39. Tsuchido, T.; Katsui, N.; Takeuchi, A.; Takano, M.; Shibasaki, I. Destruction of the outer-membrane permeability barrier of Escherichia-coli by heat-treatment. Appl. Environ. Microbiol. 1985, 50, 298-303. [PubMed]

40. American National Standard Institute. American National Standard for Safe Use of Lasers; Laser Institute of America: Orlando, FL, USA, 2000.

(C) 2017 by the authors; licensee MDPI, Basel, Switzerland. This article is an open access article distributed under the terms and conditions of the Creative Commons Attribution (CC-BY) license (http://creativecommons.org/licenses/by/4.0/). 